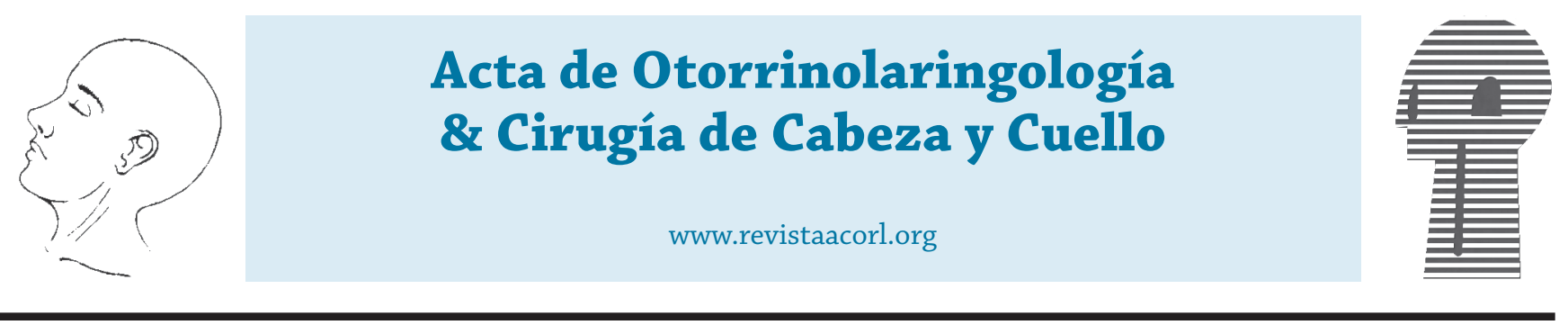

Trabajos Originales

\title{
Correlación entre los valores de escala de Epworth con índice de microdespertares en polisomnografía de pacientes con apnea de sueño
}

\section{Correlation between values of Epworth Sleep Scale with arousal index in polisomnography of sleep apnea patients}

\author{
Juan David Aguas A. ${ }^{*}$, Kevin A. Guzmán-Ortiz ${ }^{* *}$, Nelson Delgado ${ }^{* * *}$, Jorge Urzola*** \\ * Residente de otorrinolaringología, Universidad del Rosario, Bogotá. \\ ** Otorrinolaringólogo somnólogo, Hospital Militar Central UMNG, Bogotá. \\ *** Otorrinolaringólogo UMNG; Magíster Epidemiología Universidad El Bosque, Bogotá. \\ **** Médico con énfasis en salud pública, Universidad de la Sabana, Chía.
}

Forma de citar: Aguas JD,Guzman-Ortiz KA, Delgado N, Urzola J. Correlación entre los valores de escala de Epworth con índice de microdespertares en polisomnografía de pacientes con apnea de sueño.2018 a 2020. Acta otorrinolaringol. cir. cabeza cuello. 2020;48(1):46-52.Doi:516

INFORMACIÓN DEL ARTÍCULO

Historia del artículo:

Recibido: 10 de Diciembre de 2019

Evaluado: 25 de Abril de 2020

Aceptado: 21 de Mayo de 2020

Palabras clave (DeCS):

Somnolencia, Trastornos de

Somnolencia Excesiva, trastornos del

sueño, microdespertar

Correspondencia:

Juan David Aguas Agredo

Email: juan.aguas@urosario.edu.co

Dirección: Calle 37 \# 28 2 -25

Teléfono: 3508547411

\section{RESUMEN}

Introducción: el síndrome de apnea-hipoapnea obstructiva del sueño, es una enfermedad caracterizada por la obstrucción o colapso recurrente de la vía aérea superior. La escala de Epworth evalúa la somnolencia diurna subjetiva de los pacientes con un cuestionario siendo esta la más usada en la actualidad. Los microdespertares producidos durante los trastornos del sueño se ha encontrado que pueden generar somnolencia diurna excesiva. Objetivo: describir una correlación entre el índice de microdespertares y la escala de Epworth. Diseño: Corte transversal descriptivo analítico. Metodología: revisión de polisomnografías realizadas y a partir de estas obtenciones de los valores de la escala de Epworth e índice de microdespertares, describir una posible correlación entre ellos. Resultados: se evaluaron 130 pacientes y se estableció que no existía correlación estadísticamente 
significativa entre los valores de la escala de Epworth y el índice de microdespertares. Discusión: la subjetividad de la escala Epworth nos puede llevar a encontrar cierta variabilidad en los resultados de la prueba. Por otro lado, es importante tener en cuenta que hay otros aspectos que pueden generar un aumento de la somnolencia diurna medida con la escala de Epworth como lo es la hipoxemia nocturna aumentada. También es importante mencionar que existen otros elementos de la microestructura del sueño que pueden generar resultados diferentes al comparar con la definición y parámetros de microdespertares, como es el patrón alternante cíclico y sus subtipos. Conclusiones: no existe correlación entre los valores de la escala de Epworth y el índice de microdespertares.

\section{ABSTRACT}

Key words (MeSH):

Sleepiness, disorders of excessive somnolence, sleep disorder, microarousal
Introduction: Obstructive sleep apnea is a disease characterized by recurrent obstruction or collapse of the upper airway. Epworth Sleepiness Scale (ESS) assesses the subjective daytime sleepiness of patients with a questionnaire, being this the most used in nowadays. It's been found that the arousals produced during sleep disorders can develop excessive daytime sleepiness. Objective: To describe a correlation between arousal index and ESS. Design: Analytic descriptive cross-sectional study. Methodology: Review of sleep study, obtain values of ESS and arousal index and then describe a possible correlation between them. Results: 130 patients were assessed and it was established that there is no statistically significant correlation between the ESS values and the arousal index. Discussion: The subjectivity of ESS can lead to finding some variation in the tested outcomes; on the other hand, it's important to keep in mind that there are other factors that can improve the daytime sleepiness measured with ESS, such as increased nocturnal hypoxemia. Furthermore, it's convenient to identify that there are other elements of sleep microstructure that can generate different results when comparing its definition and taking arousal parameters into account, such as the cyclic alternative pattern and its subtypes. Conclusion: There is no correlation between ESS and arousal index.

\section{Introducción}

El síndrome de apnea-hipoapnea obstructiva del sueño (SAHOS), es una enfermedad que se caracteriza por la obstrucción o colapso recurrente de la vía aérea superior, con disminución del flujo de aire, hipoxemia intermitente y despertares durante el sueño (1). Es una entidad incidente y prevalente que se asocia a un alto riesgo cardiovascular y neurocognitivo que puede tener un alto impacto socioeconómico en la población (2). En Colombia, la prevalencia de los trastornos respiratorios del sueño es del $27 \%$, lo que constituye un problema de salud pública. Sin embargo, no se cuenta con datos exactos de la frecuencia de las enfermedades en el país (3). Está descrito que la prevalencia de alto riesgo de apnea del sueño en Bogotá, Bucaramanga y Santa Marta, según el cuestionario Berlín fue del 19\%, con la escala STOP BANG del 26,9\% (4). La escala de Epworth (EE) es un cuestionario simple que pide a la persona realizar una puntuación en una escala de 0 a 3 a partir de ocho situaciones cotidianas de la vida dando como rango de resultado de 0 a 24 y a partir de esta tener una medida subjetiva de la somnolencia diurna (5).

Actualmente, se ha encontrado que a un mayor puntaje en la escala de Epworth hay mayor probabilidad de presen- tar somnolencia diurna; inicialmente, un puntaje de corte de 16 descrito por Johns en 1991 fue sugerido que determinaba un "mayor nivel de somnolencia diurna" (6), posteriormente, se sugirió un corte de 10 en el puntaje y este es el punto de corte más usado actualmente (7). En este contexto, se ha encontrado que un corte de 10 o más en puntaje de la EE tiene una sensibilidad del $93,5 \%$ y una especificidad de $100 \%$ en identificar los pacientes con narcolepsia $(7,8)$. En los pacientes con SAHOS severo se ha encontrado puntajes elevados en la EE y estos puntajes tienen una relación directa con la severidad del SAHOS (5). Estos pacientes que padecen SAHOS sufren de somnolencia diurna excesiva tienen un riesgo incrementado de presentar accidentes automovilísticos, ineficiencia laboral, pobres relaciones sociales $(9,10)$.

Hay varios estudios de correlación que han intentado ver la posible causa de relación entre las características nocturnas del SAHOS y la función diaria (11-13). A pesar de estos intentos por buscar establecer esta relación no ha sido posible establecer si estas características secundarias al exceso de somnolencia diurna son debidas a fragmentación del sueño causado por microdespertares (13) o por hipoxemia intermitente nocturna asociada a trastornos respiratorios del sueño (14). Algunos estudios han podido mostrar que los microdespertares producidos en el sueño llevan a somnolencia diurna excesiva y alteración de la función diaria $(15,16)$. 
Durante el sueño cada evento respiratorio, sea apnea, hipopnea o RERA (esfuerzo respiratorio asociado a despertar), es seguido por un microdespertar el cual altera la estructura del sueño (17). Los microdespertares son un parámetro que se mide a partir de un índice en la polisomnografía, el cual se ha usado en algunos estudios a partir de la definición de Cheshire y la definición de la academia americana de medicina del sueño (AAMS) (18). Para la AAMS un microdespertar se define como un retorno a onda alfa o teta en el electroencefalograma por un periodo mínimo de 3 segundos durante la fase de sueño NMOR (no movimientos oculares rápidos); y además un aumento del tono muscular submentoniano registrado en la electromiografía para la fase de sueño MOR (movimientos oculares rápidos).

En 1995, Douglas y Mathur modifican la definición de la AAMS estableciendo una duración de criterio mínima de 1,5 segundos. Cheshire establece un microdespertar como un retorno a onda alfa o teta en el electroencefalograma por un periodo mínimo de 1,5 segundos acompañado de un aumento del tono en la electromiografía. Todas estas tres definiciones deben tener un periodo mínimo de sueño de al menos 10 segundos previo al registro del microdespertar (18). Generalmente, según esta base teórica, se puede plantear una hipótesis, siendo esta: ¿a mayor número de microdespertares en el registro polisomnográfico habrá una puntuación mayor en la escala de Epworth?

¿Puedo sospechar o crear una impresión de magnitud de anormalidad en el índice de los microdespertares en la polisomnografía con el puntaje obtenido en la escala de Epworth?

\section{Objetivos}

\section{General}

Describir si existe una correlación demostrable entre el índice de microdespertares y el puntaje de la escala de Epworth en un grupo de pacientes a quien se les realizó polisomnografía en un hospital de tercer nivel en Bogotá entre el año 2018 a 2020.

\section{Específicos}

Describir los grados de somnolencia diurna dada por un puntaje en la escala de Epworth en los pacientes a los que se les evaluó dicha escala previa realización de polisomnografía en el periodo de tiempo evaluado.

Describir estadísticamente las características sociodemográficas de los pacientes a los que se les realizó polisomnografía en el periodo de tiempo evaluado, al identificar el género más común y el grupo de edad más frecuente.

\section{Metodología}

Se realizó una revisión retrospectiva de un grupo de polisomnografías realizadas en un hospital de tercer nivel en Colombia en el año 2018, 2019 y 2020 en pacientes que presentaban sospecha de síndrome de apnea-hipoapnea del sueño. A partir de esta revisión se obtuvieron los valores de la escala de Epworth la cual fue obtenida previa realización de polisomnografía y el índice de microdespertares.

Los criterios de inclusión para esta selección fueron pacientes con sospecha de SAHOS que fueron llevados a polisomnografía. Los criterios de exclusión fueron narcolepsia, hipersomnolencia, síndrome de fatiga crónica, desordenes psiquiátricos o neurológicos. El equipo usado para el registro y análisis fue el "Embla". El equipo de laboratorio de somnología del hospital está equipado con 9 estaciones de registro polisomnográfico y la lectura de las polisomnografías fue realizada por el equipo de neurología y somnólogos de dicho centro de salud. El registro y análisis de polisomnografía fue realizado mediante el software Embla Remlogic Software 3.4. La duración de cada polisomnografía fue de aproximadamente 7 horas en promedio.

El índice de microdespertares fue tomado según la clasificación de la AASM en el cual cada índice de microdespertares fue definido como la suma del número de cada despertar por hora de tiempo total de sueño $(19,20)$. Se realizó una descripción de las características de las polisomnografías específicamente describiendo los valores del índice de microdespertares mediante frecuencia, porcentajes, media y desviación estándar en los casos de variables cuantitativas con distribución normal. Las variables numéricas con distribución normal; en este caso, ambas, tanto índice de microdespertares y puntuación de escala de Epworth, se describió con medianas y rangos intercuartílicos.

Se realizó un análisis bivariado para comparar los diferentes grupos de escala de Epworth e índices de microdespertares. Todos los datos necesarios para la investigación se recolectaron y tabularon en una tabla de Excel y se evaluaron estadísticamente con el programa SPSS v23 (Chicago, Illinois USA), con el cual se realizó un análisis estadístico descriptivo. Se calculó si existía distribución normal de los datos a través de la prueba de Kolgomorov-Smirnov. Finalmente, posterior al planteamiento de hipótesis, se aplicó prueba paramétrica de correlación de Pearson.

\section{Aspectos y consideraciones éticas}

Esta investigación se aprueba por el comité de ética respectivo. Para dicho estudio no se utilizaron datos sensibles que pudieran generar identificación o exposición de información confidencial de los pacientes; solo se usaron las variables de edad, género y parámetros polisomnográficos.

\section{Resultados}

Se tomaron un total de 130 pacientes, de los cuales fueron 90 $(69 \%)$ hombres y 40 (31\%) mujeres con una media de edad de 50,6 \pm 15 años con un rango de 18 a 90 años. Las Figuras 1 y 2 muestran las características sociodemográficas de los pacientes evaluados; se describe edad y sexo en asociación al puntaje de la EE e índice de microdespertares. Los datos de edad presentan una distribución normal con una $\mathrm{p}=0,000318$ 
$(\mathrm{p}<0,05)$. Los datos recolectados sobre la EE presentan una distribución normal con una $p=0,015(p<0,05)$. Así mismo, los datos recolectados sobre los microdespertares presentaron una distribución normal con una $\mathrm{p}=0,0000018(\mathrm{p}<0,05)$.

Dentro de nuestras opciones de hipótesis planteadas encontramos como hipótesis nula (Ho): no hay correlación entre microdespertares y puntaje en la EE; e hipótesis alterna (Ha): si hay correlación entre microdespertares y puntaje en la EE con un nivel de significancia estipulado: 0,05 (5\%). En los resultados, que según esto planteado nos dieron las pruebas de correlación, encontramos que fue de $-0,044$ con una significancia estadística de $p=0,621$. Lo cual no permite rechazar la hipótesis nula. No se encontró correlación lineal estadísticamente significativa evidenciable entre los microdespertares y el puntaje en la EE, como lo evidencia la Figura 3 y la Tabla 1, donde se evidencia el resultado encontrado en la correlación de Pearson.

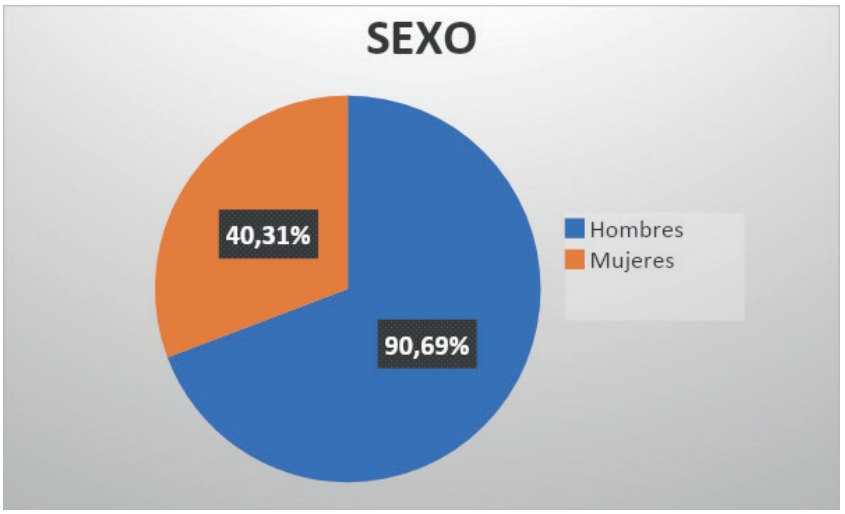

Figura 1. Distribución por género (Texto en figura:

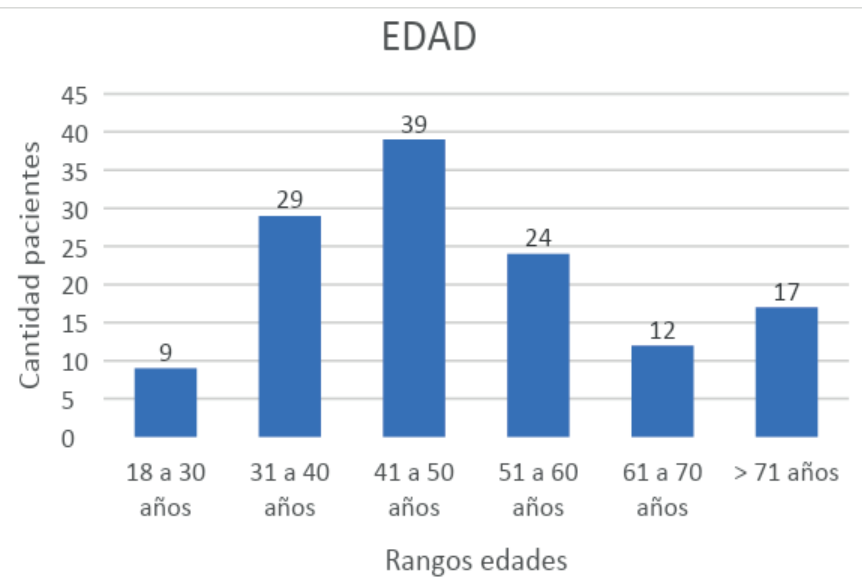

Figura 2. Número de pacientes según rangos de edad

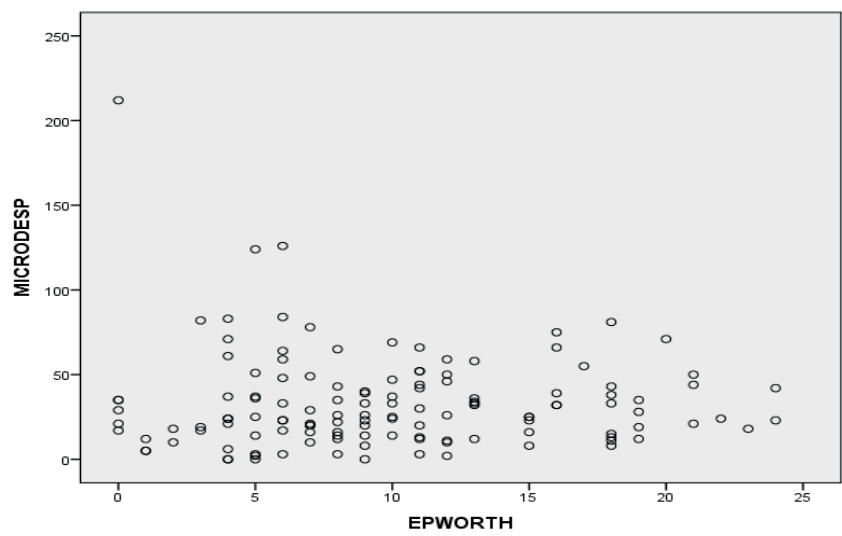

Figura 3. Correlación entre puntaje de escala Epworth e índice de microdespertares

Tabla 1. Correlaciones Epworth y Microdespertares

\begin{tabular}{|c|l|c|c|}
\hline \multicolumn{2}{|c|}{ Correlaciones } \\
\hline \multirow{3}{*}{ Epworth } & Correlación de Pearson & Epworth & Microdespertares \\
\cline { 2 - 4 } & Sig. (bilateral) & 1 & $-0,044$ \\
\cline { 2 - 4 } & $\mathrm{N}$ & 130 & 0,621 \\
\hline \multirow{3}{*}{ Microdespertares } & Correlación de Pearson & $-0,044$ & 130 \\
\cline { 2 - 4 } & Sig. (bilateral) & 0,621 & 1 \\
\cline { 2 - 4 } & $\mathrm{N}$ & 130 & 130 \\
\hline
\end{tabular}

\section{Discusión}

Algunos estudios han intentado encontrar correlaciones entre los valores de los parámetros polisomnográficos y el exceso de somnolencia diurna o con el puntaje de la EE $(11,20$, 21). Investigaciones como la realizada por Bausmer U. han podido demostrar una correlación débil entre los valores de índice de microdespertares y el puntaje de EE (21). Otras, como la realizada por Seneviratne U. y Sun Y. han evidenciado un aumento significativo en el índice de microdespertares, donde comparan pacientes exceso de somnolencia diurna con pacientes sin exceso de somnolencia diurna $(22,23)$. Mientras, otras no han encontrado ningún tipo de correlación estadísticamente significativa entre los pacientes con somnolencia diurna en exceso y sin somnolencia diurna en exceso con los índices de microdespertares $(24,25)$.

Nuestro estudio no encontró una correlación estadísticamente significativa entre los valores de índice de microdespertares y el puntaje de la EE aplicada a los pacientes, previa realización de polisomnografía; lo que deja 
la imposibilidad de descartar la hipótesis nula previamente planteada. Desde el punto de vista teórico surgen varias inquietudes que pueden presentarse secundarias a estos hallazgos y los resultados encontrados, en comparación con los que se evidenciaron en los reportes previamente descritos. Debemos recordar que, como lo mencionan algunos estudios, el exceso de somnolencia diurna en un paciente con SAHOS puede deberse ya sea a fragmentación del sueño que impide una consolidación del mismo alterando la arquitectura normal del sueño $(21,26)$ o a hipoxemia nocturna. La cual se considera un factor importante y determinante, ya que en algunos estudios se ha evidenciado en pacientes con apnea del sueño severa un empeoramiento progresivo de la hipoxemia nocturna dados por el nadir de oxígeno registrado en la polisomnografía $(20,23,27)$. A pesar de que los mecanismos biológicos no son muy bien conocidos, se cree que puede deberse a un daño neuronal severo, secundario a la hipoxemia aumentada, que lleva a daño en las estructuras promotoras de la vigila lo que conduce a somnolencia diurna excesiva (28).

Teniendo en cuenta estos resultados y esta comparación con la literatura encontrada pensamos que una de las causas que pudo llevar a que los resultados no fueran similares a los encontrados en la literatura donde sí hubo relación estadísticamente significativa entre el índice de microdespertares y el puntaje de la EE es el método en el cual se evaluó la somnolencia diurna de los pacientes y el carácter subjetivo que tiene esta EE; mientras los estudios donde se encontró una correlación estadísticamente significativa usaron un método más objetivo de la somnolencia diurna excesiva denominado el test de latencia múltiple del sueño $(22,23)$.

Solo uno de los estudios, el realizado por Bausmer Uta describe una relación débilmente positiva entre el índice de microdespertares y el puntaje de la EE. Estos resultados pueden deberse al método y el carácter subjetivo que presenta la EE, ya que ésta siempre depende de la estructura de personalidad, así como de factores emocionales más no de una medición objetiva de esa somnolencia diurna excesiva (29). Se han descrito además estudios donde se menciona una correlación entre el puntaje de la EE con varios cuestionarios para depresión (30), lo que genera factores de confusión que lleven a la persona que responde el cuestionario a tener un resultado erróneo. Consideramos que a pesar que no se encontró una correlación entre los índices de microdespertares y los puntajes de la EE, vale la pena realizar un número mayor de investigaciones mediante métodos más objetivos que permitan describir correlaciones y confirmar o descartar la existencia de las mismas entre los índices de microdespertares y la somnolencia diurna excesiva; ya que, como lo describimos previamente, aún no se tiene claridad estadísticamente significativa si estos niveles dependen exclusivamente de la fragmentación del sueño o de la hipoxemia nocturna.

Nuestro estudio nos muestra una ausencia de esta correlación entre las dos variables indicando, además, que mediante la medida subjetiva de somnolencia diurna con la aplicación de la EE no tendrá ningún tipo de correlación con el índice de microdespertares siempre y cuando estemos buscando medir la somnolencia diurna de un paciente. Sería interesante realizar un estudio similar donde se pueda medir la somnolencia diurna con una medida más objetiva y tener en cuenta otros parámetros como la saturación de oxígeno.

Por otro lado, hay que tener en cuenta que existen otros elementos de la microestructura del sueño que no son tenidos en cuenta en los manuales de estratificación de la AAMS, como es el patrón alternante cíclico, que habla de una fase A y B con unos subtipos A1, A2 y A3. Se ha encontrado que el tipo de subtipos de fase A se correlaciona con la estabilidad del sueño y los procesos cognitivos y se ha descrito que el subtipo A1 presenta una mejor sincronía electroencefalográfica durante el sueño donde además con mecanismos cerebrales involucrados en la etapa de sueño NMOR (no movimientos oculares rápidos) que se han visto relacionados con mejor desempeño cognitivo; por otro lado el tipo A2 y A3 presentan la mayoría de sus despertares en sueño NMOR (no movimientos oculares rápidos) donde en general se encuentran el $95 \%$ de pacientes con subtipo A3 y $62 \%$ de pacientes con subtipo A2 que cumplen los criterios de la AASM para microdespertares (31).

Estos subtipos se han visto en relación con un funcionamiento neurocognitivo alterado donde específicamente se ha encontrado al subtipo A2 con bajo rendimiento cognitivo y el A3 con planeamiento y secuenciación motora negativa (32). La amplia superposición entre este subtipo A2 y A3 está relacionada con la edad y su correlación con la cantidad de sueño NMOR ligero y negativa correlación con la cantidad de sueño NMOR profundo (31). De esta forma consideramos que existen otros aspectos sobre la microestructura del sueño que deben ser considerados como el patrón alternante cíclico y sus subtipos, ya que estos se consideran la principal expresión de la microestructura del sueño y pueden determinar la categorización la misma a partir de estos (32), lo que genera una posibilidad de investigación para tener en cuenta sobre la correlación entre la microestructura del sueño a partir de estos subtipos de patrón de alternancia cíclica del sueño, los microdespertares y el nivel de somnolencia diurna excesiva.

\section{Conclusiones}

- En el estudio realizado no se encontró una correlación entre el puntaje obtenido en la escala de Epworth de los pacientes evaluados con su índice de microdespertares obtenido a partir de la polisomnografía; por lo que sería un buen punto de partida, teniendo en cuenta lo descrito, investigar si se debe tener en cuenta otros factores que modifican la microestructura del sueño, fragmentación del sueño; o por el contrario la somnolencia diurna sea secundaria a un problema de hipoxemia nocturna crónica.

- Son variados los resultados obtenidos en la literatura que intentan encontrar algún tipo de correlación entre las variables evaluadas en nuestra investigación donde 
algunos encuentran resultados débilmente positivos y otros ningún tipo de correlación.

- El uso del método subjetivo para medición de somnolencia diurna excesiva mediante la escala de Epworth puede generar resultados erróneos por tratarse de una prueba que ha estado relacionada con factores emocionales asociados y puede alterarse con trastornos de personalidad por lo que tomaría mayor validez y objetividad realizarse una prueba mucho más objetiva.

- Se deben tener en cuenta otros aspectos descritos acerca de la microestructura del sueño como los subtipos de patrón alternante cíclico que generan diferentes formas de expresión de los microdespertares y así generar diferentes resultados acerca de esta correlación.

\section{Conflicto de interés}

Los autores declaramos no tener ningún conflicto de interés para el desarrollo del presente estudio.

\section{Financiación del proyecto}

Los autores declaran que este proyecto no recibió financiación.

\section{REFERENCIAS}

1. Venegas-Mariño M, García JC. Pathophysiology of obstructive sleep apnea-hypopnea syndrome (OSAHS). Rev Fac Med. 2017;65(1):S25-8.

2. Redline S, Young T. Epidemiology and natural history of obstructive sleep apnea. Ear, Nose Throat J. 1993;72(1):20$21+24$.

3. Ruiz A, Sepúlveda MAR, Martínez PH, Muñoz MC, Mendoza LO, Centanaro OPP, et al. Prevalence of sleep complaints in Colombia at different altitudes. Sleep Sci [Internet]. 2016;9(2):100-5. Disponible en: http://dx.doi.org/10.1016/j. slsci.2016.05.008

4. Hidalgo-Martínez P, Lobelo R. Global, latin-american and colombian epidemiology and mortality by obstructive sleep apnea-hypopnea syndrome (OSAHS). Rev Fac Med. 2017;65(1):S17-20.

5. Johns MW. Daytime sleepiness, snoring, and obstructive sleep apnea; The Epworth Sleepiness Scale. Chest [Internet]. 1993;103(1):30-6. Disponible en: http://dx.doi.org/10.1378/ chest.103.1.30

6. Johns MW. A new method for measuring daytime sleepiness: The Epworth sleepiness scale. Sleep. 1991;14(6):540-5.

7. Johns MW. Sleepiness in Different Situations Measured by the Epworth Sleepiness Scale. Sleep. 1994;17(8):703-10.

8. M.W.Johns. Sensitivity and specificity of the multiple sleep latency test (MSLT), the maintenance of wakefulness test and the Epworth sleepiness scale: Failure of the MSLT as a gold standard. J Sleep Res. 2001;9(1):5-11.

9. Neher RE. Letters to the Editor. Soil Horizons. 1974;15(2):5.

10. Kales A, Caldwell AB, Cadieux RJ, Vela-bueno A, Ruch LG, Mayes SD. SEVERE OBSTRUCTIVE SLEEP APNEA-II : ASSOCIATED AND CONSEQUENCES. 1985;38(5):427-34.
11. Roure N, Gomez S, Mediano O, Duran J, Peña M de la, Capote F, et al. Daytime sleepiness and polysomnography in obstructive sleep apnea patients. Sleep Med. 2008;9(7):727-31.

12. Greenberg GD, Watson RK, Deptula D. Neuropsychological dysfunction in sleep apnea. Sleep. 1987;10(3):254-62.

13. Roehrs T, Zorick F, Wittig R, Conway W, Roth T. Predictors of objective level of daytime sleepiness in patients with sleeprelated breathing disorders. Chest [Internet]. 1989;95(6):12026. Disponible en: http://dx.doi.org/10.1378/chest.95.6.1202

14. Bedard MA, Montplaisir J, Richer F, Malo J. Nocturnal hypoxemia as a determinant of vigilance impairment in sleep apnea syndrome. Chest. 1991;100(2):367-70.

15. Stepanski EJ. The effect of sleep fragmentation on daytime function. Sleep. 2002;25(3):268-76.

16. Philip P, Stoohs R, Guilleminault C. Short Note Sleep Fragmentation in Normals : A Model for Sleepiness Associated With Upper Airway Resistance Syndrome. 2018;17(July):242-7.

17. Colt HG, Haas H, Rich GB. Hypoxemia vs sleep fragmentation as cause of excessive daytime sleepiness in obstructive sleep apnea. Chest [Internet]. 1991;100(6):1542-8. Disponible en: http://dx.doi.org/10.1378/chest.100.6.1542

18. Martin SE, Engleman HM, Kingshott RN, Douglas NJ. Microarousals in patients with sleep apnoea/hypopnoea syndrome. J Sleep Res. 1997;6(4):276-80.

19. Bonnet MH, Carley DW, Carskadon MA, Easton PA. EEG arousals: scoring rules and examples: a preliminary report from the Sleep Disorders Atlas Task Force of the American Sleep Disorders Association. Sleep [Internet]. 1992;15(2):173-84. Disponible en: http://www.ncbi.nlm.nih.gov/pubmed/11032543

20. Lee SJ, Kang HW, Lee LH. The relationship between the Epworth Sleepiness Scale and polysomnographic parameters in obstructive sleep apnea patients. Eur Arch Oto-RhinoLaryngology. 2012;269(4):1143-7.

21. Bausmer U, Gouveris H, Selivanova O, Goepel B, Mann W. Correlation of the Epworth Sleepiness Scale with respiratory sleep parameters in patients with sleep-related breathing disorders and upper airway pathology. Eur Arch Oto-RhinoLaryngology. 2010;267(10):1645-8.

22. Seneviratne U, Puvanendran K. Excessive daytime sleepiness in obstructive sleep apnea: Prevalence, severity, and predictors. Sleep Med. 2004;5(4):339-43.

23. Sun Y, Ning Y, Huang L, Lei F, Li Z, Zhou G, et al. Polysomnographic characteristics of daytime sleepiness in obstructive sleep apnea syndrome. Sleep Breath. 2012;16(2):375-81.

24. Mediano O, Barceló A, de la Peña M, Gozal D, Agustí A, Barbé F. Daytime sleepiness and polysomnographic variables in sleep apnoea patients. Eur Respir J. 2007;30(1):110-3.

25. Kapur VK, Baldwin CM, Resnick HE, Gottlieb DJ, Nieto FJ. Sleepiness in Patients with Moderate to Severe SleepDisordered Breathing. Sleep. 2005;28(4):472-8.

26. Stepanski E, Lamphere J, Badia P, Zorick F, Roth T. Sleep fragmentation and daytime sleepiness. Sleep. 1984;7(1):18-26.

27. Chen R, Xiong KP, Lian YX, Huang JY, Zhao MY, Li JX, et al. Daytime sleepiness and its determining factors in Chinese obstructive sleep apnea patients. Sleep Breath. 2011;15(1):12935.

28. Veasey SC, Davis CW, Fenik P, Zhan G, Hsu YJ, Pratico D, et al. Long-term intermittent hypoxia in mice: Protracted 
hypersomnolence with oxidative injury to sleep-wake brain regions. Sleep. 2004;27(2):194-201.

29. Hayashida K, Inoue Y, Chiba S, Yagi T, Urashima M, Honda Y, et al. Factors influencing subjective sleepiness in patients with obstructive sleep apnea syndrome. Psychiatry Clin Neurosci. 2007;61(5):558-63.

30. Olson LG, Cole MF, Ambrogetti A. Correlations among Epworth Sleepiness Scale scores, multiple sleep latency tests and psychological symptoms. J Sleep Res. 1998;7(4):248-53.

31. Terzano MG, Parrino L, Rosa A, Palomba V, Smerieri A. CAP and arousals in the structural development of sleep: An integrative perspective. Sleep Med. 2002;3(3):221-9.

32. Parrino L, Ferri R, Bruni O, Terzano MG. Cyclic alternating pattern ( CAP ): The marker of sleep instability. Sleep Med Rev [Internet]. 2012;16(1):27-45. Disponible en: http://dx.doi. org/10.1016/j.smrv.2011.02.003 